\title{
Ostrowski-type fractional integral inequalities for mappings whose derivatives are $h$-convex via Katugampola fractional integrals
}

\author{
Ghulam Farid, Udita N. Katugampola and Muhammad Usman
}

\begin{abstract}
In this paper we generalize some Riemann-Liouville fractional integral inequalities of Ostrowski-type for $h$-convex functions via Katugampola fractional integrals, generalizations of the Riemann-Liouville and the Hadamard fractional integrals. Also we deduce some known results by using $p$-functions, convex functions and $s$-convex functions.
\end{abstract}

Mathematics Subject Classification (2010): 26A33, 26A51, 26D07, 26D10, 26D15.

Keywords: Ostrowski inequality, fractional integrals, convex functions, $h$-Convex functions.

\section{Introduction}

The following inequality is known as Ostrowski inequality [17] (see also, [16, page 468]) which gives an upper bound for approximation of the integral average by the value $f(x)$ at a point $x \in[a, b]$. It is proved by Ostrowski in 1938 .

Theorem 1.1. Let $f: I \rightarrow \mathbb{R}$, where $I$ is an interval in $\mathbb{R}$, be a differentiable mapping in $I^{\circ}$, the interior of $I$ and $a, b \in I^{\circ}, a<b$. If $\left|f^{\prime}(t)\right| \leq M$ for all $t \in[a, b]$, then we have

$$
\left|f(x)-\frac{1}{(b-a)} \int_{a}^{b} f(t) d t\right| \leq\left[\frac{1}{4}+\frac{\left(x-\frac{a+b}{2}\right)^{2}}{(b-a)^{2}}\right](b-a) M,
$$

where $x \in[a, b]$.

Ostrowski and Ostrowski-type inequalities have great importance in numerical analysis as they provide the bounds of different quadrature rules [1]. Over the years researchers are working to obtain Ostrowski-type inequalities for different kinds of functions. Recently Ostrowski-type inequalities via Riemann-Liouville fractional integrals are in focus (see $[3,4,5,6,14,15]$ and references therein). 
Definition 1.2. A function $f$ is called convex function on the interval $[a, b]$ if for any two points $x, y \in[a, b]$ and any $t$, where $0 \leq t \leq 1$,

$$
f(t x+(1-t) y) \leq t f(x)+(1-t) f(y) .
$$

Definition 1.3. [2] A non-negative function $f: I \rightarrow \mathbb{R}$ is said to be $p$-function, if for any two points $x, y \in I$ and $t \in[0,1]$,

$$
f(t x+(1-t) y) \leq f(x)+f(y) .
$$

Definition 1.4. [7] A function $f: I \rightarrow \mathbb{R}$ is said to be Godunova-Levin function, if for any two points $x, y \in I$ and $t \in(0,1)$,

$$
f(t x+(1-t) y) \leq \frac{f(x)}{t}+\frac{f(y)}{1-t} .
$$

$s$-convex functions in the second sense have been introduced by Hudzik and Maligranda in [10] as follows.

Definition 1.5. [10] A function $f:[0, \infty) \rightarrow \mathbb{R}$ is called $s$-convex in the second sense on the interval $[0, \infty)$ if for any two points $x, y \in[0, \infty)$ and any $t$ where $0 \leq t \leq 1$ and for some fixed $s \in(0,1]$,

$$
f(t x+(1-t) y) \leq t^{s} f(x)+(1-t)^{s} f(y) .
$$

Definition 1.6. [19] Let $J \subseteq \mathbb{R}$ be an interval containing $(0,1)$ and let $h: J \rightarrow \mathbb{R}$ be a positive function. We say $f: I \rightarrow \mathbb{R}$ is a $h$-convex function, if $f$ is non-negative and

$$
f(t x+(1-t) y) \leq h(t) f(x)+h(1-t) f(y)
$$

for all $x, y \in I$ and $t \in(0,1)$. If above inequality is reversed, then $f$ is called $h$-concave.

It is easy to see that

(i) If $h(t)=t$, then (1.1) gives non-negative convex function.

(ii) If $h(t)=\frac{1}{t}$, then (1.1) gives Godunova-levin function.

(iii) If $h(t)=1$, then (1.1) gives $p$-function.

(iv) If $h(t)=t^{s}$ where $s \in(0,1)$, then (1.1) gives $s$-convex function in the second sense.

In a paper by Sonin in 1869 [18], he used the Cauchy's integral formula as a starting point to reach the differentiation with arbitrary index. Letnikov [13] extended the idea of Sonin a short time later in 1872. Both tried to define fractional derivatives by utilizing a closed contour. Finally, Laurent in [12] used a contour given as an open circuit instead of a closed circuit, led to the definition of the Riemann-Liouville fractional integral, which is due to a little known paper published by Holmgren in $1865[9]$.

Definition 1.7. [12] Let $f \in L_{1}[a, b]$. The Riemann-Liouville fractional integrals $J_{a+}^{\alpha} f$ and $J_{b-}^{\alpha} f$ of order $\alpha>0$ with $a \geq 0$ are defined by

$$
J_{a+}^{\alpha} f(x)=\frac{1}{\Gamma(\alpha)} \int_{a}^{x}(x-t)^{\alpha-1} f(t) d t, x>a
$$


and

where

$$
J_{b-}^{\alpha} f(x)=\frac{1}{\Gamma(\alpha)} \int_{x}^{b}(t-x)^{\alpha-1} f(t) d t, x<b,
$$

$$
\Gamma(\alpha)=\int_{0}^{\infty} e^{-u} u^{\alpha-1} d u
$$

is the integral representation of Euler gamma function. Here

$$
J_{a+}^{0} f(x)=J_{b-}^{0} f(x)=f(x) .
$$

In case of $\alpha=1$, the Riemann-Liouville fractional integrals reduces to the classical integral.

Definition 1.8. J. Hadamard introduced the Hadamard fractional integral in [8], and is given by

$$
I_{a^{+}}^{\alpha} f(x)=\frac{1}{\Gamma(\alpha)} \int_{a}^{x}\left(\log \frac{x}{\tau}\right)^{\alpha-1} f(\tau) \frac{d \tau}{\tau}
$$

for $\operatorname{Re}(\alpha)>0, x>a \geq 0$.

Recently Katugampola generalized Riemann-Liouville and Hadamard fractional integrals into a unique form as follows.

Definition 1.9. [11] Let $[a, b]$ be a finite interval in $\mathbb{R}$. Then the Katugampola fractional integrals of order $\alpha>0$ for a real valued function $f$ are defined by

$$
{ }^{\rho} I_{a+}^{\alpha} f(x)=\frac{\rho^{1-\alpha}}{\Gamma(\alpha)} \int_{a}^{x} t^{\rho-1}\left(x^{\rho}-t^{\rho}\right)^{\alpha-1} f(t) d t
$$

and

$$
{ }^{\rho} I_{b-}^{\alpha} f(x)=\frac{\rho^{1-\alpha}}{\Gamma(\alpha)} \int_{x}^{b} t^{\rho-1}\left(t^{\rho}-x^{\rho}\right)^{\alpha-1} f(t) d t
$$

with $a<x<b$ and $\rho>0$, if the integrals exist, where $\Gamma(\alpha)$ is the Euler gamma function. For $\rho=1$, Katugampola fractional integrals give Riemann-Liouville fractional integrals, while $\rho \rightarrow 0^{+}$produces the Hadamard fractional integral. For its proof one can refer [11].

We organize the paper as follows:

In this paper we prove some Ostrowski-type inequalities for mappings whose derivatives are $h$-convex via Katugampola fractional integrals. We deduce some known results by using $p$-functions, convex functions and $s$-convex functions. In particular we find Ostrowski-type inequalities for Riemann-Liouville fractional integrals.

\section{Ostrowski-type fractional inequalities for $h$-convex functions via Katugampola fractional integral}

In this section we present some Ostrowski-type inequalities for $h$-convex functions via Katugampola fractional integrals. The following lemma is very useful to obtain our results. 
Lemma 2.1. Let $f:\left[a^{\rho}, b^{\rho}\right] \rightarrow \mathbb{R}$ be a differentiable mapping on $\left(a^{\rho}, b^{\rho}\right)$ with $a<b$ such that $f^{\prime} \in L_{1}[a, b]$. Then we have the following equality

$$
\begin{aligned}
& f\left(x^{\rho}\right)-\frac{(\alpha \rho+\rho-1) \Gamma(\alpha)}{\rho^{1-\alpha}}\left[\frac{\rho_{x_{x}^{-}}^{\alpha} f\left(a^{\rho}\right)}{2\left(x^{\rho}-a^{\rho}\right)^{\alpha}}+\frac{\rho I_{x^{+}}^{\alpha} f\left(b^{\rho}\right)}{2\left(b^{\rho}-x^{\rho}\right)^{\alpha}}\right] \\
& =\frac{\rho\left(x^{\rho}-a^{\rho}\right)}{2} \int_{0}^{1} t^{\alpha \rho+\rho-1} f^{\prime}\left(t^{\rho} x^{\rho}+\left(1-t^{\rho}\right) a^{\rho}\right) d t \\
& -\frac{\left(b^{\rho}-x^{\rho}\right)}{2} \int_{0}^{1} t^{\alpha \rho+\rho-1} f^{\prime}\left(t^{\rho} x^{\rho}+\left(1-t^{\rho}\right) b^{\rho}\right) d t ; x \in(a, b),
\end{aligned}
$$

with $\alpha, \rho>0$.

Proof. It is easy to see that

$$
\begin{aligned}
& \int_{0}^{1} t^{\alpha \rho+\rho-1} f^{\prime}\left(t^{\rho} x^{\rho}+\left(1-t^{\rho}\right) a^{\rho}\right) d t \\
& =\left.\frac{t^{\alpha \rho+\rho-1} f\left(t^{\rho} x^{\rho}+\left(1-t^{\rho}\right) a^{\rho}\right)}{\rho t^{\rho-1}\left(x^{\rho}-a^{\rho}\right)}\right|_{0} ^{1} \\
& -\frac{\alpha \rho+\rho-1}{\rho\left(x^{\rho}-a^{\rho}\right)} \int_{0}^{1} t^{\alpha \rho-1} f\left(t^{\rho} x^{\rho}+\left(1-t^{\rho}\right) a^{\rho}\right) d t \\
& =\frac{f\left(x^{\rho}\right)}{\rho\left(x^{\rho}-a^{\rho}\right)}-\frac{\alpha \rho+\rho-1}{\rho\left(x^{\rho}-a^{\rho}\right)} \int_{a}^{x}\left(\frac{y^{\rho}-a^{\rho}}{x^{\rho}-a^{\rho}}\right)^{\alpha-1} \frac{y^{\rho-1} f\left(y^{\rho}\right)}{x^{\rho}-a^{\rho}} d y \\
& =\frac{f^{\rho}\left(x^{\rho}\right)}{\rho\left(x^{\rho}-a^{\rho}\right)}-\frac{\rho I_{x^{-}}^{\alpha}\left(a^{\rho}\right)(\alpha \rho+\rho-1) \Gamma(\alpha)}{\rho^{2-\alpha}\left(x^{\rho}-a^{\rho}\right)^{\alpha+1}}
\end{aligned}
$$

and

$$
\begin{aligned}
& \int_{0}^{1} t^{\alpha \rho+\rho-1} f^{\prime}\left(t^{\rho} x^{\rho}+\left(1-t^{\rho}\right) b^{\rho}\right) d t \\
& =\left.\frac{t^{\alpha \rho+\rho-1} f\left(t^{\rho} x^{\rho}+\left(1-t^{\rho}\right) b^{\rho}\right)}{\rho t^{\rho-1}\left(x^{\rho}-b^{\rho}\right)}\right|_{0} ^{1} \\
& -\frac{\alpha \rho+\rho-1}{\rho\left(x^{\rho}-b^{\rho}\right)} \int_{0}^{1} t^{\alpha \rho-1} f\left(t^{\rho} x^{\rho}+\left(1-t^{\rho}\right) b^{\rho}\right) d t \\
& =\frac{-f\left(x^{\rho}\right)}{\rho\left(b^{\rho}-x^{\rho}\right)}+\frac{\alpha \rho+\rho-1}{\rho\left(b^{\rho}-x^{\rho}\right)} \int_{x}^{b}\left(\frac{y^{\rho}-b^{\rho}}{x^{\rho}-b^{\rho}}\right)^{\alpha-1} \frac{y^{\rho-1} f\left(y^{\rho}\right)}{x^{\rho}-b^{\rho}} d y \\
& =\frac{-f\left(x^{\rho}\right)}{\rho\left(b^{\rho}-x^{\rho}\right)}+\frac{I_{x^{+}}^{\alpha} f\left(b^{\rho}\right)(\alpha \rho+\rho-1) \Gamma(\alpha)}{\rho^{2-\alpha}\left(b^{\rho}-x^{\rho}\right)^{\alpha+1}} .
\end{aligned}
$$

Multiplying (2.2) by $\frac{\rho\left(x^{\rho}-a^{\rho}\right)}{2}$ and $(2.3)$ by $\frac{\rho\left(b^{\rho}-x^{\rho}\right)}{2}$, then adding resulting equations we get $(2.1)$.

Theorem 2.2. Let $f:\left[a^{\rho}, b^{\rho}\right] \subseteq[0, \infty) \rightarrow \mathbb{R}$ be a differentiable mapping on $\left(a^{\rho}, b^{\rho}\right)$ with $a<b$ such that $f^{\prime} \in L_{1}[a, b]$. If $\left|f^{\prime}\right|$ is $h$-convex on $\left[a^{\rho}, b^{\rho}\right]$ and $\left|f^{\prime}\left(x^{\rho}\right)\right| \leq M$, 
$x \in[a, b]$, then the following inequality for Katugampola fractional integrals holds

$$
\begin{aligned}
& \left|f\left(x^{\rho}\right)-\frac{(\alpha \rho+\rho-1) \Gamma(\alpha)}{\rho^{1-\alpha}}\left[\frac{{ }^{\rho} I_{x^{-}}^{\alpha} f\left(a^{\rho}\right)}{2\left(x^{\rho}-a^{\rho}\right)^{\alpha}}+\frac{{ }^{\rho} I_{x^{+}}^{\alpha} f\left(b^{\rho}\right)}{2\left(b^{\rho}-x^{\rho}\right)^{\alpha}}\right]\right| \\
& \leq \frac{M \rho\left(b^{\rho}-a^{\rho}\right)}{2} \int_{0}^{1} t^{\alpha \rho+\rho-1}\left[h\left(t^{\rho}\right)+h\left(1-t^{\rho}\right)\right] d t ; x \in(a, b),
\end{aligned}
$$

with $\alpha, \rho>0$.

Proof. Using Lemma 2.1, $h$-convexity of $\left|f^{\prime}\right|$, and upper bound of $\left|f^{\prime}\left(x^{\rho}\right)\right|$ we have

$$
\begin{aligned}
& \left|f\left(x^{\rho}\right)-\frac{(\alpha \rho+\rho-1) \Gamma(\alpha)}{\rho^{1-\alpha}}\left[\frac{\rho}{2\left(x^{\rho}-a^{\rho}\right)^{\alpha}}+\frac{\rho^{\rho} I_{x^{+}}^{\alpha} f\left(b^{\rho}\right)}{2\left(b^{\rho}-x^{\rho}\right)^{\alpha}}\right]\right| \\
& \leq \frac{\rho\left(x^{\rho}-a^{\rho}\right)}{2} \int_{0}^{1} t^{\alpha \rho+\rho-1}\left|f^{\prime}\left(t^{\rho} x^{\rho}+\left(1-t^{\rho}\right) a^{\rho}\right)\right| d t \\
& +\frac{\rho\left(b^{\rho}-x^{\rho}\right)}{2} \int_{0}^{1} t^{\alpha \rho+\rho-1}\left|f^{\prime}\left(t^{\rho} x^{\rho}+\left(1-t^{\rho}\right) b^{\rho}\right)\right| d t \\
& \leq \frac{\rho\left(x^{\rho}-a^{\rho}\right)}{2} \int_{0}^{1} t^{\alpha \rho+\rho-1}\left[h\left(t^{\rho}\right)\left|f^{\prime}\left(x^{\rho}\right)\right|+h\left(1-t^{\rho}\right)\left|f^{\prime}\left(a^{\rho}\right)\right|\right] d t \\
& +\frac{\rho\left(b^{\rho}-x^{\rho}\right)}{2} \int_{0}^{1} t^{\alpha \rho+\rho-1}\left[h\left(t^{\rho}\right)\left|f^{\prime}\left(x^{\rho}\right)\right|+h\left(1-t^{\rho}\right)\left|f^{\prime}\left(b^{\rho}\right)\right|\right] d t \\
& \leq \frac{M \rho\left(x^{\rho}-a^{\rho}\right)}{2} \int_{0}^{1} t^{\alpha \rho+\rho-1}\left[h\left(t^{\rho}\right)+h\left(1-t^{\rho}\right)\right] d t \\
& +\frac{M \rho\left(b^{\rho}-x^{\rho}\right)}{2} \int_{0}^{1} t^{\alpha \rho+\rho-1}\left[h\left(t^{\rho}\right)+h\left(1-t^{\rho}\right)\right] d t \\
& =\frac{M \rho\left(b^{\rho}-a^{\rho}\right)}{2} \int_{0}^{1} t^{\alpha \rho+\rho-1}\left[h\left(t^{\rho}\right)+h\left(1-t^{\rho}\right)\right] d t .
\end{aligned}
$$

This completes the proof.

Corollary 2.3. In Theorem 2.2 , if we take $h(t)=1$, which means that $\left|f^{\prime}\right|$ is $p$-function, then (2.4) becomes the following inequality

$$
\begin{aligned}
& \left|f\left(x^{\rho}\right)-\frac{(\alpha \rho+\rho-1) \Gamma(\alpha)}{\rho^{1-\alpha}}\left[\frac{{ }^{\rho} I_{x^{-}}^{\alpha} f\left(a^{\rho}\right)}{2\left(x^{\rho}-a^{\rho}\right)^{\alpha}}+\frac{{ }^{\rho} I_{x^{+}}^{\alpha} f\left(b^{\rho}\right)}{2\left(b^{\rho}-x^{\rho}\right)^{\alpha}}\right]\right| \\
& \leq \frac{M\left(b^{\rho}-a^{\rho}\right)}{\alpha+1} ; x \in[a, b],
\end{aligned}
$$

with $\alpha, \rho>0$.

Remark 2.4. (i) If we put $\rho=1$ in (2.4) we get [15, Theorem 1].

(ii) If we put $\rho=1, \alpha=1$ and $x=\frac{a+b}{2}$ in (2.4) we get [15, Corollary 3].

(iii) If we put $\rho=1$ and $h(t)=t$, which means that $\left|f^{\prime}\right|$ is convex function in (2.4), then we get $[15$, Corollary 1$]$. 
(iv) If we put $\rho=1$ and $h(t)=t^{s}$, which means that $\left|f^{\prime}\right|$ is $h$-convex function in (2.4), then we get [15, Corollary 2].

Theorem 2.5. Let $f:\left[a^{\rho}, b^{\rho}\right] \subseteq[0, \infty) \rightarrow \mathbb{R}$ be a differentiable mapping on $\left(a^{\rho}, b^{\rho}\right)$ with $a<b$ such that $f^{\prime} \in L_{1}[a, b]$. If $\left|f^{\prime}\right|^{q}, q>1$, is h-convex on $\left[a^{\rho}, b^{\rho}\right]$ and $\left|f^{\prime}\left(x^{\rho}\right)\right| \leq M$, $x \in[a, b]$, then the following inequality for Katugampola fractional integrals holds

$$
\begin{aligned}
& \left|f\left(x^{\rho}\right)-\frac{(\alpha \rho+\rho-1) \Gamma(\alpha)}{\rho^{1-\alpha}}\left[\frac{{ }^{\rho} I_{x^{-}}^{\alpha} f\left(a^{\rho}\right)}{2\left(x^{\rho}-a^{\rho}\right)^{\alpha}}+\frac{{ }^{\rho} I_{x^{+}}^{\alpha} f\left(b^{\rho}\right)}{2\left(b^{\rho}-x^{\rho}\right)^{\alpha}}\right]\right| \\
& \leq \frac{M \rho\left(b^{\rho}-a^{\rho}\right)}{2(p(\alpha \rho+\rho-1)+1)^{\frac{1}{p}}}\left(\int_{0}^{1}\left[h\left(t^{\rho}\right)+h\left(1-t^{\rho}\right)\right] d t\right)^{\frac{1}{q}} ; x \in(a, b),
\end{aligned}
$$

with $\alpha, \rho>0$ and $\frac{1}{p}+\frac{1}{q}=1$.

Proof. Using Lemma 2.1 and Holder's inequality we have

$$
\begin{aligned}
& \left|f\left(x^{\rho}\right)-\frac{(\alpha \rho+\rho-1) \Gamma(\alpha)}{\rho^{1-\alpha}}\left[\frac{\rho I_{x^{-}}^{\alpha} f\left(a^{\rho}\right)}{2\left(x^{\rho}-a^{\rho}\right)^{\alpha}}+\frac{\rho_{x^{+}}^{\alpha} f\left(b^{\rho}\right)}{2\left(b^{\rho}-x^{\rho}\right)^{\alpha}}\right]\right| \\
& \leq \frac{\rho\left(x^{\rho}-a^{\rho}\right)}{2} \int_{0}^{1} t^{\alpha \rho+\rho-1}\left|f^{\prime}\left(t^{\rho} x^{\rho}+\left(1-t^{\rho}\right) a^{\rho}\right)\right| d t \\
& +\frac{\rho\left(b^{\rho}-x^{\rho}\right)}{2} \int_{0}^{1} t^{\alpha \rho+\rho-1}\left|f^{\prime}\left(t^{\rho} x^{\rho}+\left(1-t^{\rho}\right) b^{\rho}\right)\right| d t \\
& \leq \frac{\rho\left(x^{\rho}-a^{\rho}\right)}{2}\left(\int_{0}^{1} t^{p(\alpha \rho+\rho-1)} d t\right)^{\frac{1}{p}}\left(\int_{0}^{1}\left|f^{\prime}\left(t^{\rho} x^{\rho}+\left(1-t^{\rho}\right) a^{\rho}\right)\right|^{q} d t\right)^{\frac{1}{q}} \\
& +\frac{\rho\left(b^{\rho}-x^{\rho}\right)}{2}\left(\int_{0}^{1} t^{p(\alpha \rho+\rho-1)} d t\right)^{\frac{1}{p}}\left(\int_{0}^{1}\left|f^{\prime}\left(t^{\rho} x^{\rho}+\left(1-t^{\rho}\right) a^{\rho}\right)\right|^{q} d t\right)^{\frac{1}{q}} .
\end{aligned}
$$

Since $\left|f^{\prime}\right|^{q}$ is $h$-convex and $\left|f^{\prime}\left(x^{\rho}\right)\right| \leq M, x \in[a, b]$, therefore we have for $x \in(a, b)$

$$
\begin{aligned}
\left|f\left(x^{\rho}\right)-\frac{(\alpha \rho+\rho-1) \Gamma(\alpha)}{\rho^{1-\alpha}}\left[\frac{\rho I_{x}^{\alpha} f\left(a^{\rho}\right)}{2\left(x^{\rho}-a^{\rho}\right)^{\alpha}}+\frac{\rho^{\alpha} I_{x^{+}} f\left(b^{\rho}\right)}{2\left(b^{\rho}-x^{\rho}\right)^{\alpha}}\right]\right| \\
\leq \frac{\rho\left(x^{\rho}-a^{\rho}\right)}{2}\left(\int_{0}^{1} t^{p(\alpha \rho+\rho-1)} d t\right)^{\frac{1}{p}}\left(\int_{0}^{1}\left[h\left(t^{\rho}\right)\left|f^{\prime}(x)\right|^{q}+h\left(1-t^{\rho}\right)\left|f^{\prime}\left(a^{\rho}\right)\right|^{q}\right] d t\right)^{\frac{1}{q}} \\
+\frac{\rho\left(b^{\rho}-x^{\rho}\right)}{2}\left(\int_{0}^{1} t^{p(\alpha \rho+\rho-1)} d t\right)^{\frac{1}{p}}\left(\int_{0}^{1}\left[h\left(t^{\rho}\right)\left|f^{\prime}(x)\right|^{q}+h\left(1-t^{\rho}\right)\left|f^{\prime}\left(b^{\rho}\right)\right|^{q}\right] d t\right)^{\frac{1}{q}} \\
\leq \frac{M \rho\left(x^{\rho}-a^{\rho}\right)}{2 p(\alpha \rho+\rho-1)+1)^{\frac{1}{p}}}\left(\int_{0}^{1}\left[h\left(t^{\rho}\right)+h\left(1-t^{\rho}\right)\right] d t\right)^{\frac{1}{q}} \\
+\frac{M \rho\left(b^{\rho}-x^{\rho}\right)}{2(p(\alpha \rho+\rho-1)+1)^{\frac{1}{p}}}\left(\int_{0}^{1}\left[h\left(t^{\rho}\right)+h\left(1-t^{\rho}\right)\right] d t\right)^{\frac{1}{q}}
\end{aligned}
$$




$$
=\frac{M \rho\left(b^{\rho}-a^{\rho}\right)}{2(p(\alpha \rho+\rho-1)+1)^{\frac{1}{p}}}\left(\int_{0}^{1}\left[h\left(t^{\rho}\right)+h\left(1-t^{\rho}\right)\right] d t\right)^{\frac{1}{q}} .
$$

This completes the proof.

Corollary 2.6. In Theorem 2.5, if we take $h(t)=1$, which means that $\left|f^{\prime}\right|^{q}$ is $p$ function, then (2.6) becomes the following inequality

$$
\begin{aligned}
& \left|f\left(x^{\rho}\right)-\frac{(\alpha \rho+\rho-1) \Gamma(\alpha)}{\rho^{1-\alpha}}\left[\frac{\rho}{2\left(x_{x}^{\alpha}-a^{\rho}\right)^{\alpha}}+\frac{{ }^{\rho} I_{x}^{\alpha} f\left(b^{\rho}\right)}{2\left(b^{\rho}-x^{\rho}\right)^{\alpha}}\right]\right| \\
& \leq \frac{(2)^{\frac{1}{q}-1} M \rho\left(b^{\rho}-a^{\rho}\right)}{(p(\alpha \rho+\rho-1)+1)^{\frac{1}{p}}} ; x \in[a, b],
\end{aligned}
$$

with $\alpha, \rho>0$ and $\frac{1}{p}+\frac{1}{q}=1$.

Remark 2.7. (i) If we put $\rho=1$ in (2.6) we get [15, Theorem 2].

(ii) If we put $\rho=1, \alpha=1$ and $x=\frac{a+b}{2}$ in (2.6) we get [15, Corollary 6].

(iii) If we put $\rho=1$ and $h(t)=t$ in $(2.6)$, which means that $\left|f^{\prime}\right|$ is convex function, then we get [15, Corollary 4].

(iv) If we put $\rho=1$ and $h(t)=t^{s}$, which means that $\left|f^{\prime}\right|$ is $h$-convex function in (2.6), then we get [15, Corollary 5].

Theorem 2.8. Let $f:\left[a^{\rho}, b^{\rho}\right] \subseteq[0, \infty) \rightarrow \mathbb{R}$ be a differentiable mapping on $\left(a^{\rho}, b^{\rho}\right)$ such that $f^{\prime} \in L_{1}[a, b]$, where $a<b$. If $\left|f^{\prime}\right|^{q}, q>1$ is $h$-convex on $\left[a^{\rho}, b^{\rho}\right]$ and $\left|f^{\prime}\left(x^{\rho}\right)\right| \leq M$, $x \in[a, b]$, then the following inequality for Katugampola fractional integrals holds for $x \in(a, b)$

$$
\begin{aligned}
& \left|f\left(x^{\rho}\right)-\frac{(\alpha \rho+\rho-1) \Gamma(\alpha)}{\rho^{1-\alpha}}\left[\frac{\rho^{\rho} I_{x^{-}}^{\alpha} f\left(a^{\rho}\right)}{2\left(x^{\rho}-a^{\rho}\right)^{\alpha}}+\frac{\rho^{\rho} I_{x^{+}}^{\alpha} f\left(b^{\rho}\right)}{2\left(b^{\rho}-x^{\rho}\right)^{\alpha}}\right]\right| \\
& \leq \frac{M \rho\left(b^{\rho}-a^{\rho}\right)}{2}\left(\frac{1}{\rho(\alpha+1)}\right)^{1-\frac{1}{q}}\left(\int_{0}^{1} t^{\alpha \rho+\rho-1}\left[h\left(t^{\rho}\right)+h\left(1-t^{\rho}\right)\right] d t\right)^{\frac{1}{q}},
\end{aligned}
$$

with $\alpha, \rho>0$.

Proof. Using Lemma 2.1 and power mean inequality we have

$$
\begin{gathered}
\left|f\left(x^{\rho}\right)-\frac{(\alpha \rho+\rho-1) \Gamma(\alpha)}{\rho^{1-\alpha}}\left[\frac{{ }^{\rho} I_{x^{-}}^{\alpha} f\left(a^{\rho}\right)}{2\left(x^{\rho}-a^{\rho}\right)^{\alpha}}+\frac{{ }^{\rho} I_{x^{+}}^{\alpha} f\left(b^{\rho}\right)}{2\left(b^{\rho}-x^{\rho}\right)^{\alpha}}\right]\right| \\
\leq \frac{\rho\left(x^{\rho}-a^{\rho}\right)}{2} \int_{0}^{1} t^{\alpha \rho+\rho-1}\left|f^{\prime}\left(t^{\rho} x^{\rho}+\left(1-t^{\rho}\right) a^{\rho}\right)\right| d t \\
+\frac{\rho\left(b^{\rho}-x^{\rho}\right)}{2} \int_{0}^{1} t^{\alpha \rho+\rho-1}\left|f^{\prime}\left(t^{\rho} x^{\rho}+\left(1-t^{\rho}\right) b^{\rho}\right)\right| d t \\
\leq \frac{\rho\left(x^{\rho}-a^{\rho}\right)}{2}\left(\int_{0}^{1} t^{\alpha \rho+\rho-1} d t\right)^{1-\frac{1}{q}}\left(t^{\alpha \rho+\rho-1} \int_{0}^{1}\left|f^{\prime}\left(t^{\rho} x^{\rho}+\left(1-t^{\rho}\right) a^{\rho}\right)\right|^{q} d t\right)^{\frac{1}{q}}
\end{gathered}
$$




$$
+\frac{\rho\left(b^{\rho}-x^{\rho}\right)}{2}\left(\int_{0}^{1} t^{\alpha \rho+\rho-1} d t\right)^{1-\frac{1}{q}}\left(\int_{0}^{1} t^{\alpha \rho+\rho-1}\left|f^{\prime}\left(t^{\rho} x^{\rho}+\left(1-t^{\rho}\right) a^{\rho}\right)\right|^{q} d t\right)^{\frac{1}{q}} .
$$

Since $\left|f^{\prime}\right|^{q}$ is $h$-convex and $\left|f^{\prime}\left(x^{\rho}\right)\right| \leq M, x \in[a, b]$, there for we have for $x \in(a, b)$

$$
\begin{aligned}
& \left|f\left(x^{\rho}\right)-\frac{(\alpha \rho+\rho-1) \Gamma(\alpha)}{\rho^{1-\alpha}}\left[\frac{{ }^{\rho} I_{x^{-}}^{\alpha}\left(a^{\rho}\right)}{2\left(x^{\rho}-a^{\rho}\right)^{\alpha}}+\frac{\rho^{\rho} I_{x^{+}}^{\alpha} f\left(b^{\rho}\right)}{2\left(b^{\rho}-x^{\rho}\right)^{\alpha}}\right]\right| \\
& \leq \frac{\rho\left(x^{\rho}-a^{\rho}\right)}{2}\left(\frac{1}{\rho(\alpha+1)}\right)^{1-\frac{1}{q}} \times \\
& \left(\int_{0}^{1} t^{\alpha \rho+\rho-1}\left[h\left(t^{\rho}\right)\left|f^{\prime}(x)\right|^{q}+h\left(1-t^{\rho}\right)\left|f^{\prime}\left(a^{\rho}\right)\right|^{q}\right] d t\right)^{\frac{1}{q}} \\
& +\frac{\rho\left(b^{\rho}-x^{\rho}\right)}{2}\left(\frac{1}{\rho(\alpha+1)}\right)^{1-\frac{1}{q}} \times \\
& \left(\int_{0}^{1} t^{\alpha \rho+\rho-1}\left[h\left(t^{\rho}\right)\left|f^{\prime}(x)\right|^{q}+h\left(1-t^{\rho}\right)\left|f^{\prime}\left(b^{\rho}\right)\right|^{q}\right] d t\right)^{\frac{1}{q}} \\
& \leq \frac{M \rho\left(x^{\rho}-a^{\rho}\right)}{2}\left(\frac{1}{\rho(\alpha+1)}\right)^{1-\frac{1}{q}}\left(\int_{0}^{1} t^{\alpha \rho+\rho-1}\left[h\left(t^{\rho}\right)+h\left(1-t^{\rho}\right)\right] d t\right)^{\frac{1}{q}} \\
& +\frac{M \rho\left(b^{\rho}-x^{\rho}\right)}{2}\left(\frac{1}{\rho(\alpha+1)}\right)^{1-\frac{1}{q}}\left(\int_{0}^{1} t^{\alpha \rho+\rho-1}\left[h\left(t^{\rho}\right)+h\left(1-t^{\rho}\right)\right] d t\right)^{\frac{1}{q}} \\
& =\frac{M \rho\left(b^{\rho}-a^{\rho}\right)}{2}\left(\frac{1}{\rho(\alpha+1)}\right)^{1-\frac{1}{q}}\left(\int_{0}^{1} t^{\alpha \rho+\rho-1}\left[h\left(t^{\rho}\right)+h\left(1-t^{\rho}\right)\right] d t\right)^{\frac{1}{q}} .
\end{aligned}
$$

This completes the proof.

Remark 2.9. (i) If we put $\rho=1$ in (2.8) we get [15, Theorem 3].

(ii) If we put $\rho=1, \alpha=1$ and $x=\frac{a+b}{2}$ in (2.8), we get [15, Corollary 9].

(iii) If we put $\rho=1$ and $h(t)=t$ in (2.8) which means that $\left|f^{\prime}\right|$ is convex function, then we get $[15$, Corollary 7$]$.

(iv) If we put $\rho=1$ and $h(t)=t^{s}$, which means that $\left|f^{\prime}\right|$ is $h$-convex function in (2.8), then we get [15, Corollary 8].

Corollary 2.10. In Theorem 2.8, if we take $h(t)=1$, which means that $\left|f^{\prime}\right|^{q}$ is $p$ function, then (2.8) becomes the following inequality

$$
\begin{aligned}
& \left|f\left(x^{\rho}\right)-\frac{(\alpha \rho+\rho-1) \Gamma(\alpha)}{\rho^{1-\alpha}}\left[\frac{\rho^{\rho} I_{x^{-}}^{\alpha} f\left(a^{\rho}\right)}{2\left(x^{\rho}-a^{\rho}\right)^{\alpha}}+\frac{\rho^{\rho} I_{x^{+}}^{\alpha} f\left(b^{\rho}\right)}{2\left(b^{\rho}-x^{\rho}\right)^{\alpha}}\right]\right| \\
& \leq \frac{(2)^{\frac{1}{q}-1} M\left(b^{\rho}-a^{\rho}\right)}{\rho(\alpha+1)} ; x \in(a, b),
\end{aligned}
$$

with $\alpha, \rho>0$. 
Conclusion. Due to the fact that the Katugampola fractional integrals are the generalizations of both the Riemann-Liouville fractional integrals and Hadamard fractional integrals, so in our paper by taking $\rho=1$ we have deduced the known results for Riemann-Liouville fractional integrals. All results proved in this research paper can also be deduced for the Hadamard fractional integrals by taking limits when parameter $\rho \rightarrow 0^{+}$.

Acknowledgement. The research work of Ghulam Farid is supported by the Higher Education Commission of Pakistan under NRPU 2016, Project No. 5421.

The research work of the second author was partially supported by the U.S. Army research office grant W911NF-15-1-0537.

\section{References}

[1] Dragomir, S.S., Ostrowski-type inequalities for Lebesgue integral: A survey of recent results, Aust. J. Math. Anal. Appl., 14(2017), no. 1, 1-287.

[2] Dragomir, S.S., Pečarić, J., Persson, L.E., Some inequalities of Hadamard type, Soochow J. Math., 21(1995), 335-341.

[3] Farid, G., Some new Ostrowski-type inequalities via fractional integrals, Int. J. Anal. App., 14(2017), no. 1, 64-68.

[4] Farid, G., Katugampola, U.N., Usman, M., Ostrowski-type fractional integral inequalities for S-Godunova-Levin functions via Katugampola fractional integrals, Open J. Math. Sci., 1(2017), no. 1, 97-110.

[5] Farid, G., Rafique, S., Rehman, A. Ur., More on Ostrowski and Ostrowski-Gruss type inequalities, Commun. Optim. Theory, 2017(2017), ArtID 15, 9 pages.

[6] Farid, G., Usman, M., Ostrowski-type k-fractional integral inequalities for MT-convex and h-convex functions, Nonlinear Funct. Anal. Appl., 22(2017), no. 3, 627-639.

[7] Godunova, E.K., Levin, V.I., Inequalities for functions of a broad class that contains convex, monotone and some other forms of functions, Numerical Mathematics and Mathematical Physics (Russian), Moskov. Gos. Ped. Inst. Moscow, (1985), 138-142.

[8] Hadamard, J., Essai sur l'etude des fonctions donnees par leur developpment de Taylor, Journal de Mathématiques Pures et Appliquées, 8(1892), 101-186.

[9] Holmgren, H.J., Om differentialkalkylen med indices of hvad nature sam heist, Kongliga Svenska Vetenskaps-Akademiens Handlinger, 5(1865-1866), no. 11, 1-83.

[10] Hudzik, H., Maligranda, L., Some remarks on s-convex functions, Aequationes Math., 48(1994), 100-111.

[11] Katugampola, U.N., New approach to generalized fractional derivatives, Bull. Math. Anal. Appl., 6(2014), no. 4, 1-15.

[12] Laurent, H., Sur le calcul des derivees a indicies quelconques, Nouv. Annales de Mathematik, 3(1884), no. 3, 240-252.

[13] Letnikov, A.V., An explanation of the concepts of the theory of differentiation with arbitrary index, Moscow Matem. Sbornik, 6(1872), 413-445.

[14] Liu, W., Ostrowski-type fractional integral inequalities for MT-convex function, Miskole Mathematical Notes, 16(2015), no. 1, 249-256. 
[15] Matloka, M., Ostrowski-type inequalities for functions whose derivatives are h-convex via fractional integrals, Journal of Scientific Research and Reports, 3(12)(2014), Article No. JSRR.2014.12.005, 1633-1641.

[16] Mitrinović, D.S., Pečarić, J., Fink, A.M., Inequalities involving functions and their integrals and derivatives, Kluwer Academic Publisher Group, Dordrecht, 53(1991).

[17] Ostrowski, A., Uber die Absolutabweichung einer differentierbaren funktion von ihrem integralmittelwert, Comment. Math. Helv., 10(1938), no. 1, 226-227.

[18] Sonin, N. Ya., On differentiation with arbitrary index, Moscow Matem. Sbornik, 6(1869), no. 1, 1-38.

[19] Varosanec, S., On h-convexity, J. Math. Anal. Appl., 326(2007), no. 1, 303-311.

Ghulam Farid

COMSATS University Islamabad

Attock Campus, Pakistan

e-mail: faridphdsms@hotmail.com ghlmfarid@cuiatk.edu.pk

Udita N. Katugampola

Department of Mathematics, Florida Polytechnic University

Lakeland, FL 33805, USA

e-mail: uditanalin@yahoo.com dkatugampola@floridapoly.edu

Muhammad Usman

COMSATS University Islamabad

Attock Campus, Pakistan

e-mail: usmanmani333@gmail.com 